\title{
Model for superconductivity in optimally doped electron cuprates
}

\author{
T. H. Geballe and B. Y. Moyzhes \\ Laboratory for Advanced Materials and Department of Applied Physics Stanford University, \\ Stanford, CA 94305-4045 \\ E-mail: geballe@stanford.edu
}

Received May 29, 2001

\begin{abstract}
The very anomalous normal state properties and superconductivity of electron-doped superconducting cuprates with the $T^{*}$ structure are discussed. The literature values of high mobilities for electrons and holes are explained by the electrons and holes existing in different layers. The annealing procedures necessary for superconductivity produce oxygen-vacancy negative- $U$ pairing centers. The relative insensitivity of $T_{c}$ to the annealing condition is attributed to stabilization of the chemical potential near the middle of the narrow oxygen band formed by oxygen vacancies where the electronic entropy is large.
\end{abstract}

PACS: 74.76.Bz, 72.15.Eb, 72.15.Gd, 74.25.Fy

\section{Introduction}

Pairing interactions which are confined only to the $\mathrm{CuO}_{2}$ layers of optimally hole-doped high temperature superconducting cuprates are believed to be insufficient to account for the wide range of maximum $T_{c}$, which are found in the families of superconducting cuprates which have the highest $T_{c}$ [1]. In the present paper we extend our discussion to include the electron-doped superconducting cuprates.

While pairing interactions are considered to be exclusively in the $\mathrm{CuO}_{2}$ layers in the most widely accepted models of cuprate superconductivity, there is certainly no theoretical reason why they cannot occur elsewhere. Attractive interactions across interfaces have been discussed in the past many times, starting with Ginzburg [2] and Bardeen [3]. In their models artificial interfaces were considered.

In the cuprates nature has provided layers with well-matched interfaces, with the possibility of transferring charge (or doping) from reservoir layers which are removed from the $\mathrm{CuO}_{2}$ layers. Ternary layered perovskites and structures with even more complex unit cells grown either as single crystals, or as films might be considered to be «self organized» with layer sequencing that permits modulated doping. In order to account for the very high $T_{c}$ superconducting transitions of the cuprates which have $\mathrm{Hg}, \mathrm{Tl}$ and $\mathrm{Bi}$-based charge reservoir layers, we have suggested that those $6 S^{1}$ cations are negative $U$ centers based on well-known chemistry of these elements. These provide an effective attractive potential between electrons of opposite spin [1]. The negative $U$ center forms as a result of the cation interaction with surrounding atoms (polarization, deformation, magnetic, etc.) and internal electron correlation. In the strongly ionic limit the $6 S^{1}$ cations can disproportionate to $6 S^{0}$ and $6 S^{2}$ configurations. More realistically, they would form a narrow band based upon those configurations. The quasiparticles in this partially filled band can interact with the pair ordering in the $\mathrm{CuO}_{2}$ layers and become part of the condensate. While at this stage there is no direct proof for this idea, we believe it has merit because i) it is well known that $\mathrm{Hg}, \mathrm{Bi}$ and $\mathrm{Tl}$ form negative $U$ centers in many oxides, and ii) there is the well-documented case discovered by Chernik and Lykov [4] of superconductivity resulting when small concentrations of $\mathrm{Tl}$ are substituted for $\mathrm{Pb}$ in $\mathrm{PbTe}$. Two articles review the extensive research in Russia which shows convincingly that $\mathrm{Tl}$ (and no other dopants), forms a narrow resonant band based upon $6 S^{0}-6 S^{2}$ states inside the $\mathrm{PbTe}$ valence band which, when partially occupied, becomes superconducting [5,6]. Optimum doping of about $\sim 10^{20}$ carriers per cc results in the $T_{c}$ which range up to $2 \mathrm{~K}$. It is of interest that these quantities are comparable to those found in optimally oxygen-reduced $\mathrm{SrTiO}_{3}$. 
More experiments are needed to test the idea that negative $U$ centers enhance the superconductivity of the layered cuprates. As a first step in this direction, we refer to a model which analyzes tunneling through a barrier containing negative $U$ impurities [7] and predicts that an enhanced critical current should be observed due to pair resonant tunneling.

In the present paper we show that pairing due to negative $U$ centers may be useful as a basis for understanding the perplexing chemistry and unusual normal state properties reported in the literature of the electron-doped cuprates such as $(\mathrm{NdCe})_{2} \mathrm{CuO}_{4}(\mathrm{NCCO})$ which have the $T^{*}$ structure where a separate oxygen layer exists between the rare earth layers.

\section{Electron doped cuprates}

The synthesis of electron doped superconducting cuprates is a two step process [8]. The first step is a straightforward substitution of a tetravalent cation (Ce or $\mathrm{Th}$ ) for the trivalent rare earth, such as $\mathrm{Nd}$. The added electron which is transferred to the $\mathrm{CuO}_{2}$ layer remains weakly localized. The second step involves annealing in vacuum or an inert gas which removes a small amount of oxygen $(\sim 1$ or $2 \%$ ). The second step is essential for producing superconductivity [9] and cannot be understood in terms of simple doping or charge transfer. From chemical considerations each oxygen removed leaves two electrons in the lattice. Intuitively one might expect the increased electron doping to be the same as would be obtained by the substitution of two additional $\mathrm{Ce}^{4+}$ for $\mathrm{Nd}^{3+}$ which results only in a small decrease in resistivity. However, a much more remarkable change occurs. The transport behavior shows that mobile holes and electrons are created and that superconductivity appears below about $25 \mathrm{~K}$.

The neutron diffraction data [10] show no significant structural change results from the oxygen reduction (step 2). The only difference is that a barely detectable concentration of interstitial oxygen at the apical oxygen $\left(\mathrm{O}_{3}\right)$ site (which is not occupied in the ideal $T^{*}$ phase) is no longer detected in the reduced (superconducting) single crystal.

The profound consequences of removing $1 \%$ or so of oxygen from the lattice are not at all clear. Studies of $\left(\mathrm{Nd}_{1.85} \mathrm{Ce}_{0.15}\right) \mathrm{CuO}_{4}$, the most intensively investigated system, show that the superconducting properties are only found in a narrow concentration range for compositions $0.15 \leq \mathrm{Ce} \leq 0.18$ [9], and only after reduced oxygen annealing. After this treatment the transport properties suggest semimetallic behavior with low carrier concentration. For some samples the Hall effect changes sign with temperature [11], and the Seebeck voltage can be of either sign [12]. The positive Nernst effect is 1 to 2 orders of magnitude larger than expected for a typical Fermi liquid [12]. A large positive magnetoresistance at $60 \mathrm{~K}[12,13]$ is observed which simple considerations suggest mobilities of the order of $100 \mathrm{~cm}^{2} /(\mathrm{V} \cdot \mathrm{s})$. Smaller changes in resistivity itself with doping indicate that mobility changes may be more important than doping.

\section{Suggested model}

There have been no satisfactory models proposed to explain the transport and superconductivity $n$ type cuprates. It seems apparent to us that it will be necessary to consider interactions throughout the entire complex unit cell, much as we have argued is needed to understand the hole doped cuprates [1]. For the electron doped cuprates with the highest $T_{c}$, more definitive experiments, particularly careful measurements comparing single crystals and epitaxial films before and after step 2, are needed. At present we can offer only educated guesses as to the underlying microscopic physics and chemistry.

There is no doubt that in step 1 the Ce doping transfers electrons to the $\mathrm{CuO}_{2}$ layer. The $\mathrm{CuO}$ bond distance increases and the distance between the NdCe layer and the oxygen layer decreases [14].

\section{Normal state properties}

It takes $15 \%$ Ce doping to destroy the antiferromagnetic long-range order, after which the transport remains in the weak localization regime at low temperatures.

In step 2, oxygen vacancies are created and two electrons remain in the lattice for each oxygen removed. It is well known from the properties of reduced alkaline earth oxides that oxygen vacancies are never paramagnetic [15]. We postulate that similar behavior occurs in the reduced cuprates. That this is not unexpected follows from a model comparing the energies of singly and doubly charged impurity centers in a dielectric continuum [16].

A maximum positive magnetoresistance is expected when the electron and hole conductivities are equal. This maximum is observed approximately at the same concentration as $T_{c(\max )}$ (Ref. 12, Fig. 7). The magnitude suggests there are about $10^{20}$ carriers. If they were confined to single layers one would expect strong $2 d$ electron-hole Coulomb scattering and low mobility. 
Semimetals with high mobilities such as $\mathrm{Sb}$ and $\mathrm{Bi}$ are 3 dimensional, and have very large dielectric constants; both of these factors increase the screening. We suggest that there is a different reason for the high mobility in NCCO, namely because the mobile carriers exist in different layers. The electrons are confined to the $\mathrm{CuO}_{2}$ layers and the holes are confined to the oxygen layers where the Madelung potential favors holes. It is significant that step 2 is only effective after the long-range antiferromagnetic order is destroyed by the Ce doping. The electrons in the $\mathrm{CuO}_{2}$ layers are then less localized and may promote increased conductivity in the oxygen layers. This interaction in turn should promote delocalization in the $\mathrm{CuO}_{2}$ layers [17].

\section{Superconducting interactions}

Without the oxygen vacancies there is no superconductivity. We propose that the oxygen vacancy negative $U$ centers discussed above support superconducting pairing interactions in the oxygen layers such as $\mathrm{Hg}, \mathrm{Bi}$, and $\mathrm{Tl}$ cations do in the charge reservoir layers of the hole doped type cuprates [1]. The experiments with $\mathrm{Tl}$ in $\mathrm{PbTe}$ where the hole concentration was changed by additional doping with $\mathrm{Na}$, which is a lower lying acceptor level than Tl, show that, to obtain maximum $T_{c}$ [5], the chemical potential must be close to the middle of the narrow negative $U$ band where both occupied and unoccupied negative $U$ states are present.

This position of the chemical potential is stabilized after annealing by thermodynamics due to the highest electron density of states being near the middle of the impurity band. A somewhat related behavior has been noted in oxygen nonstoichiometric $(\mathrm{HgRe}) \mathrm{Ba}_{2} \mathrm{CuO}_{y}$ where $T_{c}$ close to $T_{c(\max )}$ after annealing is achieved independently of changes in $\mathrm{Hg}$ and Re composition [18]. The procedures for obtaining $T_{c}$ in step 2 which are relatively robust and independent of the exact annealing regime, i.e., whether done in a vacuum, or in an inert gas, or in oxygen at a more elevated temperature, can reasonably be attributed to a similar thermodynamic stabilization.

\section{Conclusion}

We have proposed a model which has the virtue of being able to explain the unusual normal state and superconducting properties of electron doped cuprate superconductors for the first time. The model has unusual features, particularly that there are mobile electrons and holes present which exist in different layers of the unit cell. Superconducting pairing interactions are believed to arise from negative $U$ center oxygen vacancies which are formed upon removing oxygen by annealing. Considerably more experimental and theoretical work is required in order to prove or disprove our model.

\section{Acknowledgment}

We are grateful for the opportunity to dedicate this short note and unconventional model to the memory of L. Shubnikov, and to be able to acknowledge the enormous contributions to physics that he made during his shortened life.

The work at Stanford has been supported in part by the Air Force Office of Scientific Research.

1. T. H. Geballe and B. Y. Moyzhes, Physica C341-348, $1821(2000)$.

2. V. L. Ginzburg, Zh. Exp. Theor. Fiz. 47, 2318 (1964).

3. D. Allender, J. Bray, and J. Bardeen, Phys. Rev. B7, 1026 (1973); Phys. Rev. B8, 4443 (1973).

4. I. A. Chernik and S. N. Lykov, Pis'ma Zh. Eksp. Teor. Fiz. 7, 94 (1981); I. A. Chernik and S. N. Lykov, Fiz. Tverd. Tela (Leningrad) 23, 1400 (1981) [Sov. Phys. Solid State 23, 817 (1981).

5. V. I. Kaidanov and Yu. I. Ravich, Sov Phys. Usp. 28, 31 (1985).

6. S. A. Nemov and Yu. I. Ravich, Phys.-Usp. 41, 735 (1998).

7. V. Oganessian, S. Kivelson, T. H. Geballe, and B. Y. Moyzhes (to be published).

8. Y. Tokura, H. Takagi, and S. Uchida, Nature 337, 345 (1989).

9. Original references are given in the extensive review by $\mathrm{J}$. Fontcuberta and L. Fahrega in: Studies of High Temperature Superconductors, vol. 16, A. Narlicar (ed.), Nova Science Publishers, Inc. (1996), p. 85. It is noteworthy that similar results are obtained for a variety of annealing procedures.

10. A. J. Schultz, J. D. Jorgenson, J. L. Peng, and R. L. Greene, Phys. Rev. B53, 5157 (1996).

11. W. Jiang, S. N. Mao, X. X. Xi, X. Jiang, J. L. Peng, T. Venkatesan, C. J. Lobb, and R. L. Greene, Phys. Rev. Lett. 73, 1291 (1994).

12. P. Fournier, X. Jiang, W. Jiang, S. N. Mao, T. Venkatesan, C. J. Lobb, and R. L. Greene, Phys. Rev. B56, 14149 (1997).

13. Non-superconducting samples show a negative magnetic resistance as expected for weak localization.

14. References to original work are cited in Ref. 9.

15. J. W. Carson, D. F. Holcomb, and H. Ru'chardt, J. Phys. Chem. Solids 12, 66 (1959).

16. B. Ya. Moizhes and S. G. Suprun, Sov. Phys. Solid State 24, 309 (1982).

17. We are unaware of direct experimental evidence, but do note that a metallic overlayer produces a detectable increase in the $T_{c}$ of a MoGe thin film. D. Ephron Thesis, Correlated Electron Tunnelling and Quantum Motion of Vortices in Disordered Model Systems, Stanford University (1996); N. Mason, private communication.

18. A. Serquis, L. Fabrega, L. Morales, A. Basset, A. Caneiro, J. Fontcuberta, S. Short, J. P. Hodges, and J. Jorgensen, Physica C341-348, 511 (2000). 